\title{
An Automatic Identification Procedure to Promote the use of FES-Cycling Training for Hemiparetic Patients
}

\author{
Emilia Ambrosini, $\mathrm{PhD}^{1,2^{*}}$; Simona Ferrante, $\mathrm{PhD}^{\mathbf{1}}$; Thomas Schauer, $\mathrm{PhD}^{\mathbf{3}}$; \\ Giancarlo Ferrigno, $\mathbf{P h D}^{1}$; Franco Molteni, $\mathbf{M D}^{\mathbf{4}}$ and Alessandra Pedrocchi, $\mathbf{P h D}^{1}$ \\ ${ }^{1}$ NeuroEngineering and Medical Robotics Laboratory, NearLab, Department of \\ Electronics, Information, and Bioengineering, Politecnico di Milano, \\ Piazza Leonardo da Vinci 32, 20133, Milano, Italy \\ ${ }^{2}$ Physical Medicine and Rehabilitation Unit, Scientific Institute of Lissone, Institute of \\ Care and Research, Salvatore Maugeri Foundation IRCCS, Lissone, Italy \\ ${ }^{3}$ Control Systems Group, Technische Universität Berlin, Einsteinufer 17, \\ D-10587 Berlin, Germany \\ ${ }^{4}$ Villa Beretta Rehabilitation Center, Valduce Hospital, Via Nazario Sauro 17, \\ 23845 Costa Masnaga, Lecco, Italy
}

Submitted September 2013. Accepted for publication June 2014.

\begin{abstract}
Cycling induced by Functional Electrical Stimulation (FES) training currently requires a manual setting of different parameters, which is a time-consuming and scarcely repeatable procedure. We proposed an automatic procedure for setting session-specific parameters optimized for hemiparetic patients. This procedure consisted of the identification of the stimulation strategy as the angular ranges during which FES drove the motion, the comparison between the identified strategy and the physiological muscular activation strategy, and the setting of the pulse amplitude and duration of each stimulated muscle. Preliminary trials on 10 healthy volunteers helped define the procedure. Feasibility tests on 8 hemiparetic patients ( 5 stroke, 3 traumatic brain injury) were performed. The procedure maximized the motor output within the tolerance constraint, identified a biomimetic strategy in 6 patients, and always lasted less than 5 minutes. Its reasonable duration and automatic nature make the procedure usable at the beginning of every training session, potentially enhancing the performance of FES-cycling training.
\end{abstract}

Keywords: Functional electrical stimulation, cycling, hemiparesis, rehabilitation, stimulation parameters

*Corresponding author: Emilia Ambrosini, NeuroEngineering And medical Robotics Laboratory, NearLab, Department of Electronics, Information, and Bioengineering, Politecnico di Milano, Piazza Leonardo da Vinci 32, 20133, Milano, Italy. Phone: (0039) 022399 9509. Fax: (0039) 022399 9003. E-mail: emilia.ambrosini@polimi.it. Other authors: simona.ferrante@polimi.it; schauer@control.tu-berlin.de; giancarlo.ferrigno@polimi.it; fmolteni@valduce.it; alessandra.pedrocchi@polimi.it 


\section{INTRODUCTION}

The use of Functional Electrical Stimulation (FES) to induce a cycling movement has been receiving increased interest in the post-stroke rehabilitation. Its feasibility and effectiveness have been shown both on post-acute [1-5] and chronic stroke patients $[6,7]$. FES-cycling training improves muscle strength [1], cycling smoothness [2], peak pedaling power [7], locomotion performance [4], and motor coordination [5]. However, to improve the clinical acceptance of FES-cycling training as well as any other trainings based on FES, the equipment needs to become easier to set up and operate [8]. In particular, FES-cycling training requires initial setting of different parameters as the stimulation strategy, i.e., the on-off crank angular ranges of muscle stimulation, the pulse amplitude and duration of each stimulated muscle, and the stimulation frequency. In clinics, these parameters are usually identified manually through a time-consuming and scarcely repeatable procedure, potentially resulting in a sub-optimal training performance.

In the design of the stimulation strategy, different approaches have been proposed in the literature. The stimulation pattern was designed via a mathematical consideration of the geometry and limb weight [9]. A forward dynamic simulation of FES-cycling was developed to investigate the influence of subject-specific parameters on stimulation patterns and power output [10]. Direct manual settings were also proposed [11,12]. These methods, although more practical, computed the stimulation ranges in a static condition without any possibility to assure repeatability and to maximize the muscle force when transferred into pedal rotation. A different approach was to derive the stimulation strategy from electromyography (EMG) data of healthy volunteers pedaling on the same cycle-ergometer [13-15]. Recently, a stimulation strategy based on the physiological muscle activation timing, called "biomimetic" [16], showed promising therapeutic benefits when applied on post-acute stroke patients $[1,4,5]$. The afferent inputs provided by FES using a biomimetic stimulation strategy are physiologically synchronized with the cycling movement, theoretically helping the patients in "reminding" how to pedal voluntarily [17]. However, to the extent of our knowledge, no studies have investigated the possibility of automatically identifying a sessionspecific biomimetic stimulation strategy to account for a number of factors such as the physiological parameters, the daily condition of the patient, the seating position, the electrodes locations, or other factors that might change between subjects and days, affecting the performance of the training.

While the stimulation strategy affects the timing of the muscle contraction, the stimulation frequency, the pulse amplitude and duration overall determine the strength of the resultant muscle contraction [17]. Many studies investigated the effects of the pulse amplitude $[18,19]$ and duration $[18,20]$ on motor unit recruitment and muscle contraction force. As a general conclusion, increasing the current amplitude produces a stronger depolarizing drive that travels deeper into the underlying tissue [19], while changing the pulse duration alters the relative recruitment of motor and sensory axons. Short pulse durations (50-400 $\mu \mathrm{s}$ ) preferentially activate motor axons, whereas longer pulse durations $(0.5-1 \mathrm{~ms})$ recruit relatively more sensory axons [20]. In order to limit fatigue and discomfort, constant low frequency stimulation resulting in smooth 
contractions at lower force levels is often used [21]. In a pilot study investigating the feasibility of FES-cycling on stroke patients [1], the authors observed that all of the patients, after getting used to FES, were able to produce a positive active torque throughout the 30-minute training with a stimulation frequency of $20 \mathrm{~Hz}$. Although it has been shown that a modulation of the stimulation frequency could be effective in reducing fatigue [22], this method cannot be exploited in clinical settings since most of the commercial stimulators used for FES-cycling do not allow modulation of the stimulation frequency during training.

In clinical environment, the muscles are usually stimulated during pre-defined crank angular ranges $[1,4,6,7]$. While setting the other parameters, muscle contraction is the primary intent of most investigators. Current amplitude is manually adjusted to obtain an optimal motor response within the subject's comfort [23], whereas the stimulation frequency and the pulse width (PW) are usually fixed a priori between $20-50 \mathrm{~Hz}$ and 200-300 $\mu \mathrm{s}$, respectively [24]. This work aimed at designing a fast, reliable, and automatic procedure to set the stimulation parameters required for FES-cycling training. This session-specific identification procedure was defined in order to maximize the exercise workload within the physiological timing. It was optimized for hemiparetic patients and to be compatible with most of the commercial stimulators. The final objective of the procedure proposed in this manuscript is to overcome the manual setting and to promote the clinical acceptance of FES-cycling training.

\section{METHODS}

\subsection{Experimental Setup}

The experimental setup consisted of a motorized cycle-ergometer (Thera-Live ${ }^{\mathrm{TM}}$, Medica Medizintechnick GmbH, Germany), a current-controlled 8-channel stimulator (Rehastim $^{\mathrm{TM}}$, Hasomed GmbH, Germany), and a multi-channel signal amplifier system for EMG recordings (Porti 32 ${ }^{\mathrm{TM}}$, TMS International BV, Enschede, The Netherlands). The ergometer was customized with strain gauges mounted on the crank arms to measure the torque signals produced by each leg during pedaling and with a shaft encoder to measure the crank angle $[25,26]$. A PC running Matlab/Simulink ${ }^{\circledR}$ under Linux acquired all signals at $1024 \mathrm{~Hz}$, controlled the stimulator, and saved the data. During the trials, the subjects sat on a chair or a wheelchair in front of the ergometer and their legs were stabilized by calf supports fixed to the pedals.

\subsection{Preliminary Trials towards the Definition of the Automatic Procedure}

Trials on 10 healthy volunteers ( 8 men and 2 women; mean \pm standard deviation: $28.4 \pm 4.4$ years of age, $75.6 \pm 11.0 \mathrm{~kg}$ in weight, $180.6 \pm 7.2 \mathrm{~cm}$ in height) were performed to help define the automatic procedure. All subjects reported having no neuromuscular or skeletal impairment. Three different sets of trials were performed: the first set aimed at identifying a session-specific stimulation strategy, the second set was designed to define the muscular activation strategy used by healthy subjects for pedaling, and, finally, the last set of trials was meant to select the best way to modulate the current amplitude and the pulse duration. These protocols were approved by the Ethical Committee of the Valduce Hospital and all of the subjects provided their written informed consent. 


\subsubsection{Identification of the Session-Specific Stimulation Strategy}

The first set of trials consisted of a 30-second warm-up of passive cycling followed by four stimulation phases, lasting 30 seconds each, during which the quadriceps and hamstrings of both legs were individually stimulated during the entire revolution.

Rectangular biphasic pulses were delivered through $50 \mathrm{~mm} \times 90 \mathrm{~mm}$ surface electrodes placed over the muscle belly. On the quadriceps, the electrodes were placed along the thigh with a $10 \mathrm{~cm}$ distance between the electrodes. The distal electrode was placed $5 \mathrm{~cm}$ above the patella, slightly to the medial side, while the proximal electrode was placed more laterally. On the hamstrings, the electrodes were placed with a $7 \mathrm{~cm}$ distance between the electrodes on the midline. The stimulation frequency was fixed at $20 \mathrm{~Hz}$ as a good compromise between maximization of the force and minimization of both fatigue and discomfort [21]. The pulse amplitude and duration were set for each muscle group at values that can elicit a visible muscle contraction, without hindering the pedaling. The current could vary between 10 and $70 \mathrm{~mA}$, while the pulse width could range between 100 and $500 \mu \mathrm{s}$. Throughout the trial, the ergometer's motor maintained a cadence of 30 RPM and subjects were asked not to contribute voluntarily to the pedaling.

The torque signals produced by the stimulation of each muscle group were analyzed to identify the crank angle interval during which the stimulation drove the motion. The active torque profile as a function of the crank angle was computed as follows:

$$
\mathrm{T}_{\mathrm{i}, \mathrm{j}}^{\mathrm{FES} \text {,active }}(\theta)=\mathrm{T}_{\mathrm{i}, \mathrm{j}}^{\mathrm{FES}}(\theta)-\mathrm{T}_{\mathrm{j}}^{\text {passive }}(\theta), \quad \text { with } \mathrm{i} \in\{\mathrm{Q}, \mathrm{H}\} \text { and } \mathrm{j} \in\{\mathrm{R}, \mathrm{L}\}
$$

where $\theta$ corresponds to the crank angle, $\mathrm{T}_{\mathrm{i}, \mathrm{j}}^{\mathrm{FES} \text {,active }}$ is the active torque profile obtained by stimulating the right or left $(R, L)$ quadriceps or hamstrings $(Q, H)$,

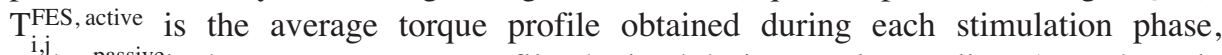
and $T_{j}^{\text {passive }}$ is the average torque profile obtained during passive cycling. A crank angle of $0^{\circ}$ corresponds to the maximal flexion of the left hip.

The static stimulation range was identified for each stimulated muscle as the angular range during which the active torque profile was greater than $25 \%$ of its peak.

\subsubsection{Identification of the Physiological Muscular Activation Strategy}

To verify whether the estimated stimulation ranges assured a biomimetic stimulation strategy, the muscular activity of healthy volunteers during volitional pedaling was analyzed.

The experimental trials consisted of a 30 -second period of passive cycling, followed by 2 minutes of voluntary pedaling. The subjects maintained exactly the same position they had during the previous trial. EMG activations of 10 muscles (rectus femoris, vastus lateralis, vastus medialis, biceps femoris caput brevis, and semitendinosus of both legs) were acquired. Surface $\mathrm{Ag} / \mathrm{AgCl}$ electrodes were placed over the target muscles following the indications of the Surface ElectroMyoGraphy for the NonInvasive Assessment of Muscles (SENIAM) project [27]. Throughout the trial, the cadence was displayed to the subjects who were asked to maintain a constant value of 30 RPM. 
The EMG recordings were analyzed to determine the timing of muscle activation during pedaling. The raw signals were high-pass filtered $\left(5^{\text {th }}\right.$ order Butterworth, cutoff frequency of $10 \mathrm{~Hz})$, rectified, and low-pass filtered $\left(5^{\text {th }}\right.$ order Butterworth filter, cutoff frequency of $5 \mathrm{~Hz}$ ). For each revolution, the EMG envelope was plotted against the crank angle by means of a cubic spline approximation, and the EMG activation profile was computed by averaging the single-revolution profiles. For each muscle, the EMG activation interval was defined as the crank angular range during which the mean EMG activation profile was greater than $25 \%$ of its peak [5]. The activation interval of the whole quadriceps was defined as the union of the activation intervals of the rectus femoris, vastus lateralis, and vastus medials; analogously, the activation interval of the hamstring was defined as the union of the activation intervals of the biceps femoris caput brevis and semitendinosus.

For each subject, the EMG activation intervals were compared to the sessionspecific stimulation ranges estimated in the previous trial. To take into account the electromechanical delay in the muscle response, the static stimulation ranges were advanced by $22^{\circ}$, which corresponds to a time delay of $120 \mathrm{~ms}$ at 30 RPM [11,28]. We refer to the phase-advanced stimulation ranges as dynamic stimulation strategy. For each muscle group, the percentage of the dynamic stimulation range covered by the EMG activation interval was computed as follows:

$$
\text { overlap }_{i, j}=\frac{\theta_{i, j}^{\text {stim }} \cap \theta_{i, j}^{\text {EMG }}}{\theta_{i, j}^{\text {stim }}}, \quad \text { with } i \in\{Q, H\} \text { and } j \in\{R, L\}
$$

where $\theta_{i, j}^{\text {stim }}$ represents the dynamic stimulation range, while $\theta_{i, j}^{\mathrm{EMG}}$ corresponds to the EMG activation interval. The overlap could range from 0 (stimulation range and EMG activation interval completely out-of-phase) to $100 \%$ (stimulation range completely covered by the EMG activation interval).

\subsubsection{Methods for Setting the Current Amplitude and the Pulse Width}

Six out of ten healthy subjects were involved in the third set of trials. These trials consisted of a 30 -second period of passive cycling followed by four stimulation phases during which the muscles were individually stimulated during the dynamic stimulation ranges previously identified. Stimulation electrodes were placed as before over the quadriceps and hamstrings of both legs. Throughout the trial, the ergometer's motor maintained a cadence of 30 RPM and subjects were asked not to voluntarily contribute to the movement. The stimulation frequency was fixed at $20 \mathrm{~Hz}$, whereas the pulse charge was increased in steps of $3.5 \mu \mathrm{C}$ every five revolutions from $0 \mu \mathrm{C}$ until reaching the pain threshold. Five different ways to distribute the same values of charge into different combinations of current amplitude and PW were compared (see Figure 1).

Each subject performed five consecutive trials to test all of the charge distributions. The execution order of the trials was randomized. To check the onset of muscular fatigue, testing pulses with fixed values of current and PW were delivered at the beginning of each trial. 

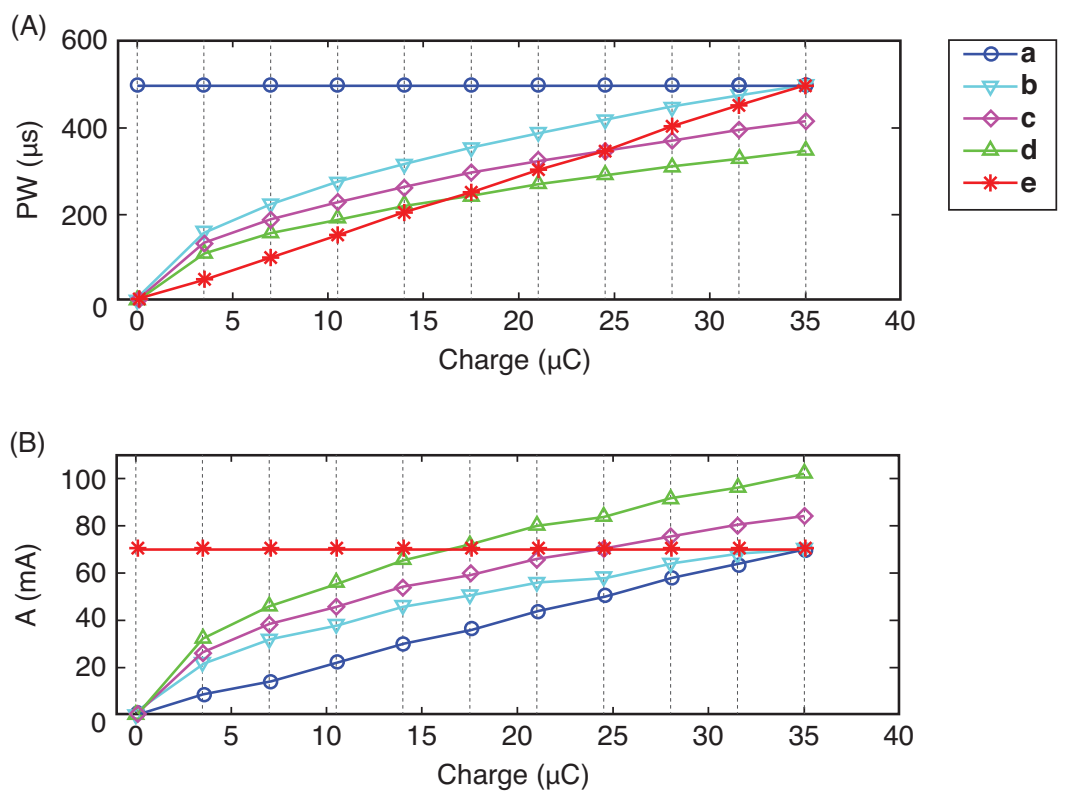

Figure 1. Charge distributions tested on healthy volunteers. At each step, the five distributions were characterized by the same value of charge differently distributed in terms of current and PW. Distribution a modulated only the current at $500 \mu \mathrm{s}$; distribution e modulated only the pulse width at $70 \mathrm{~mA}$; the other three distributions (b, c, and d) modulated both parameters.

For each revolution of the four stimulation phases, the right and left active torques were computed as shown in equation (1). The FES-induced mechanical work was then computed as follows:

$$
\mathrm{W}_{\mathrm{i}, \mathrm{j}}=\int_{\theta_{\mathrm{i}, \mathrm{j}}^{\mathrm{star}}}^{\theta_{\mathrm{si}, \mathrm{j}}^{\text {stop }}} \mathrm{T}_{\mathrm{i}}^{\mathrm{FES} \text {,active }}(\theta) \mathrm{d} \theta, \quad \text { with } \mathrm{i} \in\{\mathrm{Q}, \mathrm{H}\} \text { and } \mathrm{j} \in\{\mathrm{R}, \mathrm{L}\}
$$

where $\mathrm{W}_{\mathrm{i}, \mathrm{j}}$ represents the mechanical work induced by the stimulation of the $\mathrm{i}$-th muscle of the $\mathrm{j}$-th leg, whereas $\theta_{\mathrm{i}, \mathrm{j}}^{\text {stat }}$ and $\theta_{\mathrm{i}, \mathrm{j}}^{\text {stor }}$ are the crank angles that delimits the static stimulation range. For each muscle, the mean value of FES-induced work at each charge step was computed by averaging the values obtained during the five revolutions with a constant level of charge.

An inter-subjective statistical analysis (non-parametric Friedman test, $\mathrm{P}<0.05$ ) was carried out to compare the different charge distributions both in terms of maximal FESinduced work and tolerated charge. Post-hoc analysis (Dunn-Sidak test) was performed to determine which pairs of charge distributions were significantly different. All subjects $(\mathrm{N}=6)$ and muscles $(\mathrm{N}=4)$ not affected by muscular fatigue were included in 
the statistical analysis. Fatigue was defined as when the work values induced by the testing pulses differed more than $25 \%$.

\subsection{Definition of Identification Procedure and Feasibility Trials on Patients}

Figure 2 shows the flow chart of the automatic procedure to set the FES-cycling parameters on hemiparetic patients. The first step consisted of identifying the sessionspecific stimulation strategy. The same experimental protocol used for healthy subjects was applied, but only the muscles of the unimpaired side were stimulated. Therefore, the trial lasted for a total of 90 seconds, including 30 seconds of passive cycling, 30 seconds of stimulation of the unimpaired quadriceps, and 30 seconds of stimulation of the unimpaired hamstrings. Since the pedaling is characterized by an anti-symmetrical kinematic pattern, the stimulation ranges of the paretic muscles were obtained by introducing a $180^{\circ}$-shift of the ranges estimated for the unimpaired side.

To assure a biomimetic stimulation strategy, the estimated stimulation ranges were compared to the physiological muscular activation strategy (see Section 2.2.2). When

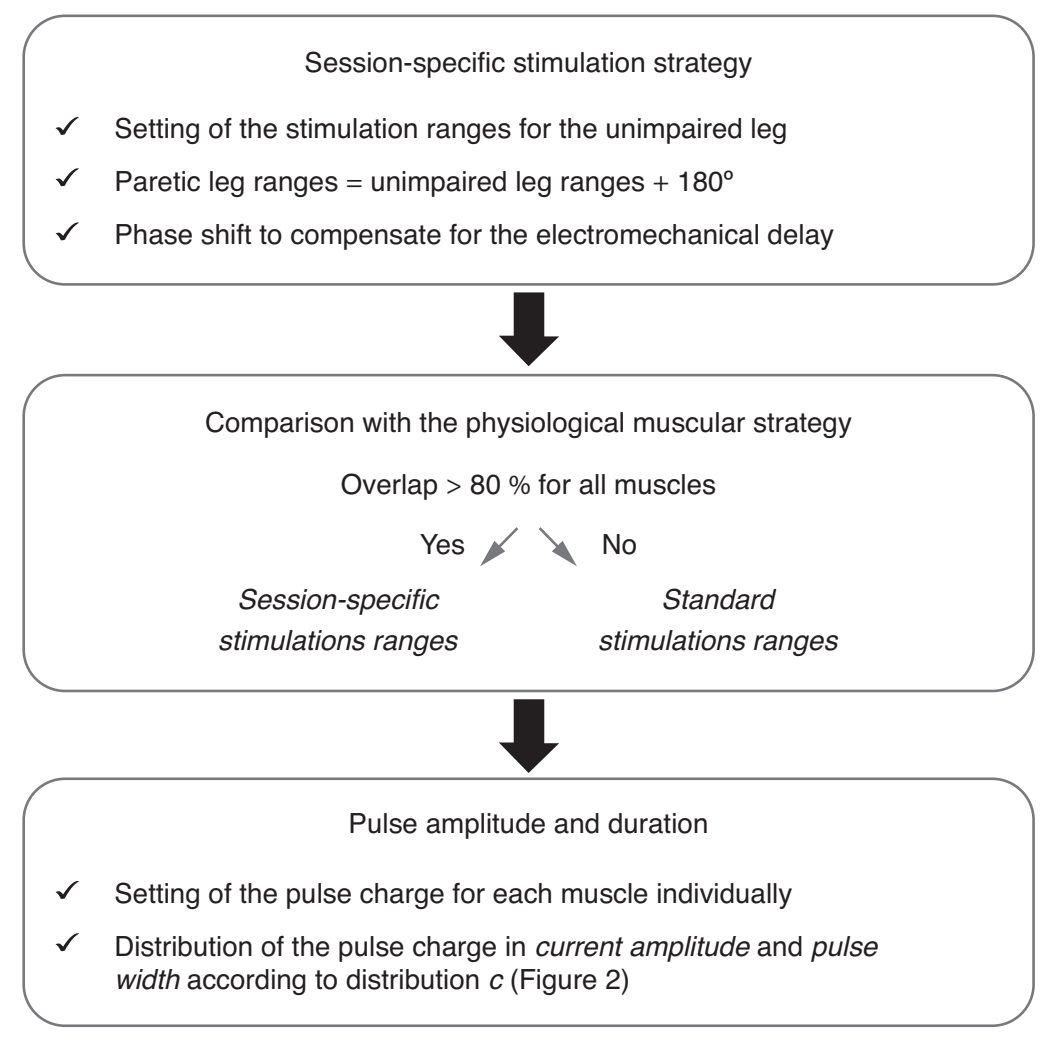

Figure 2. Experimental procedure to automatically identify FES-cycling parameters on patients. 
the stimulation strategy was biomimetic (i.e., an overlap of at least $80 \%$ was achieved for both quadriceps and hamstrings), the session-specific stimulation ranges were utilized during FES-cycling training; otherwise, standard stimulation ranges were selected. As standard stimulation ranges we used the stimulation strategy defined in [16], which was optimized starting from the EMG activations of healthy volunteers during pedaling. An overlap of $80 \%$ was taken as threshold since it represented the $5^{\text {th }}$ percentile of the overlap values achieved for the healthy subjects.

The last step consisted of setting the pulse amplitude and duration. For each muscle, the amount of charge was increased in steps of about $3.5 \mu \mathrm{C}$ every five revolutions from $0 \mu \mathrm{C}$ till reaching the pain threshold; an automated software identified the painless charge value that can induce the highest level of work at the crank. The best charge distribution identified on healthy subjects was used to divide the charge value into values of pulse amplitude and duration.

The procedure was tested on eight hemiparetic patients, whose demographic and clinical details are reported in Table 1 . All of the recruited patients had a low to mild spasticity level of the lower limb muscles (Modified Ashworth Scale $\leq 2$ ). All subjects received a detailed explanation of the study and gave their written informed consent prior to participation. The study was approved by the Ethical Committee of the Valduce Hospital.

\section{RESULTS AND DISCUSSION}

\subsection{Trials on Healthy Volunteers}

Figure 3 depicts the results of a trial to identify the stimulation strategy performed by one healthy volunteer: panels (A) and (B) show the mean torque profiles acquired at the right crank arm during passive cycling and during the stimulation phase of the right quadriceps and hamstrings, respectively; panels (C) and (D) depict the corresponding

Table 1. Demographic and clinical details of hemiparetic patients.

\begin{tabular}{|c|c|c|c|c|c|c|}
\hline Subject & $\begin{array}{c}\text { Age } \\
\text { (years) }\end{array}$ & Gender & Etiology & $\begin{array}{l}\text { Time since } \\
\text { acute event }\end{array}$ & $\begin{array}{l}\text { Affected } \\
\text { Side }\end{array}$ & $\begin{array}{c}\text { Motricity Index } \\
\text { (leg subscale) }\end{array}$ \\
\hline S1 & 66 & Female & Ischemic stroke & 2 months & Left & $58 / 100$ \\
\hline $\mathrm{S} 2$ & 58 & Female & Ischemic stroke & 6 years & Left & $64 / 100$ \\
\hline S3 & 57 & Male & Hemorrhagic stroke & $\begin{array}{c}4 \text { years \& } 7 \\
\text { months }\end{array}$ & Right & $53 / 100$ \\
\hline S4 & 65 & Male & Ischemic stroke & 1 year & Left & $43 / 100$ \\
\hline S5 & 49 & Male & Ischemic stroke & 5 months & Right & $58 / 100$ \\
\hline S6 & 74 & Male & Traumatic brain injury & $\begin{array}{l}2 \text { years \& } 1 \\
\text { month }\end{array}$ & Right & $43 / 100$ \\
\hline S7 & 46 & Male & Traumatic brain injury & $\begin{array}{c}2 \text { years \& } 4 \\
\text { months }\end{array}$ & Right & $58 / 100$ \\
\hline S8 & 39 & Male & Traumatic brain injury & $\begin{array}{c}1 \text { year \& } 1 \\
\text { month }\end{array}$ & Right & $60 / 100$ \\
\hline
\end{tabular}



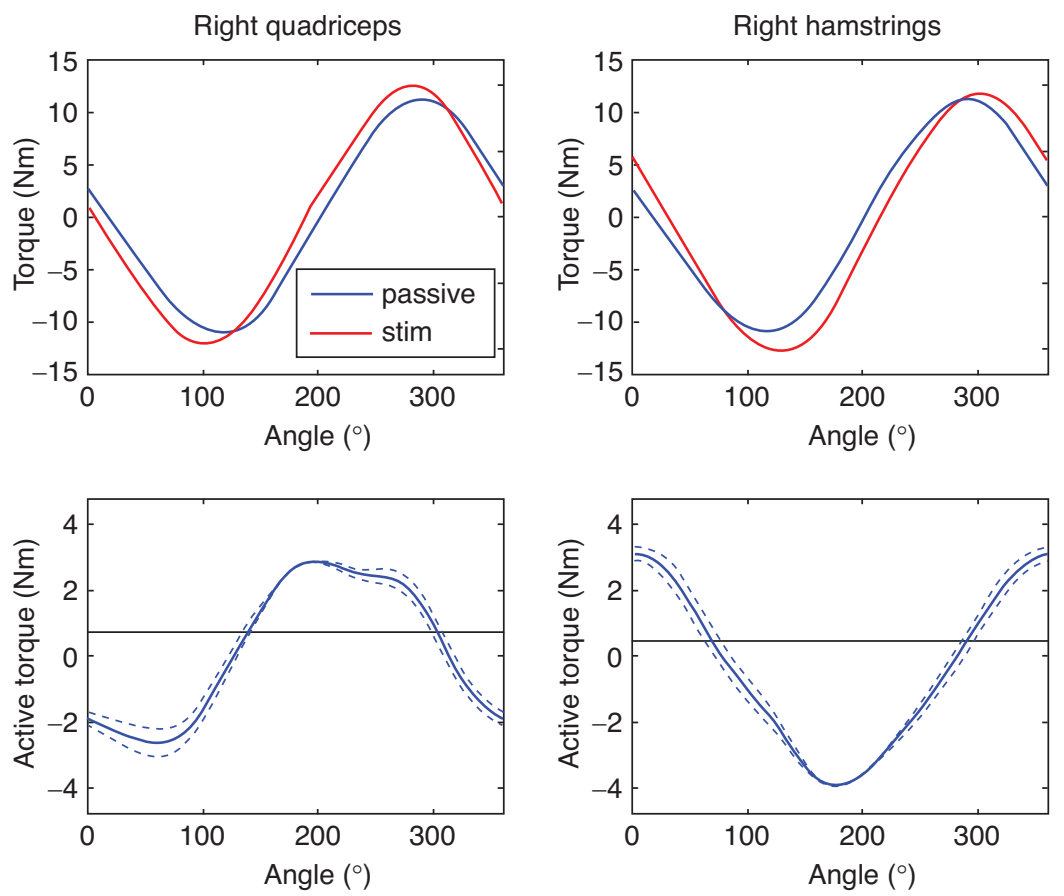

Figure 3. Results of a trial to identify the stimulation strategy on one representative healthy subject. Panels (A) and (B) depict the mean torques during passive cycling and the stimulation phases of the right quadriceps and hamstrings, respectively; panels (C) and (D) report the corresponding FES-induced active torques, with the solid line showing the mean profile, the dashed lines representing the standard deviation (SD), and the black horizontal line indicating the threshold used to identify the static stimulation ranges.

FES-induced active torque profiles. In the lower panels, the black horizontal line represents the threshold to identify the crossover angles from assisting to hindering the motion. The first $180^{\circ}$ of each revolution (i.e., $0-180^{\circ}$ ) corresponded to the push phase of the left leg and the pull phase of the right leg, vice-versa for the second $180^{\circ}$ (i.e., $\left.180-360^{\circ}\right)$. Thus, as expected, the right quadriceps (panel (C)) produced a positive active torque in the second $180^{\circ}$, whereas the positive contribution of the right hamstrings (panel (D)) straddled the two hemi-revolutions (end of the second and beginning of the first one), confirming that the hamstrings activation helps in maintaining crank progression during limb transitions [29].

During the voluntary pedaling trials, all of the healthy volunteers were able to maintain a constant cadence of 30 RPM as required. Indeed, the root mean square error between the instantaneous velocity and the nominal cadence was always less than 3.5 RPM. 


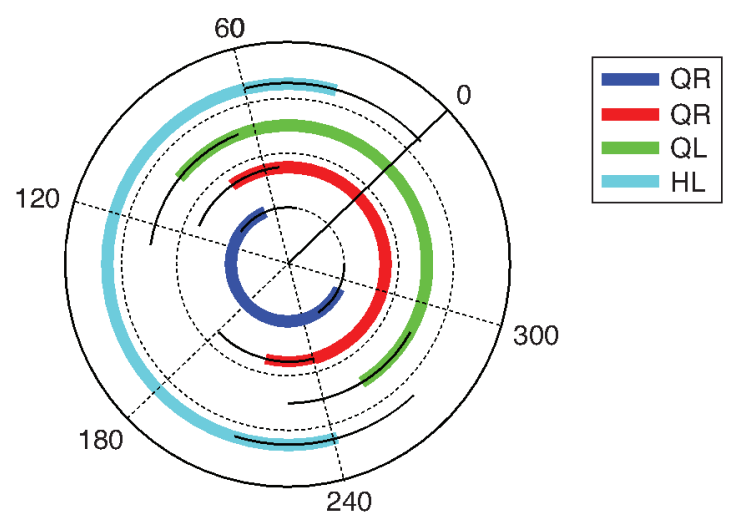

Figure 4. Muscular activation strategy adopted by healthy volunteers during pedaling as function of the crank angle. Colored arcs show the EMG activation intervals averaged among subjects, while the black arcs indicate the $\mathrm{SD}$. The $0^{\circ}$ angle corresponds to the position at which the left hip is maximally flexed. QR = right quadriceps; HR = right hamstrings; $\mathrm{QL}=$ left quadriceps; $\mathrm{HL}=$ left hamstrings.

Figure 4 shows the physiological muscular activation strategy adopted by the healthy volunteers during voluntary pedaling. The quadriceps muscles were responsible for hip flexion and knee extension, while the hamstrings muscles were responsible for hip extension and knee flexion. Homologous contralateral muscles were shifted by $180^{\circ}$, confirming that pedaling is characterized by an anti-symmetrical kinematic pattern.

The dynamic stimulation ranges (i.e., the previously identified stimulation ranges phaseadvanced to take into account the electromechanical delay) were compared to the muscular activation intervals. A median value of $100 \%$ (interquartile range of 3.8\%) was obtained in terms of overlap considering all muscles $(\mathrm{N}=4)$ and subjects $(\mathrm{N}=10)$, suggesting that the estimated stimulation ranges were completely inside the physiological activation intervals. Therefore, we could affirm that the experimental procedure was able to automatically identify a biomimetic stimulation strategy on healthy subjects.

Figure 5 compares the charge distributions in terms of maximal FES-induced work values (panel (A)) and maximal tolerated charge levels (panel (B)). Values obtained by all subjects and muscles which were not affected by muscular fatigue (14 out of 24) are reported. Although there was a high variability among subjects and muscles, a significant difference between the charge distributions in terms of work $(P=0.003)$ was found. The post-hoc analysis revealed a significant difference between distribution $\mathbf{c}$ and distributions a, d, and e. Charge distribution c obtained the maximal value of work in 8 out of 14 samples and, thus, it was selected as the solution that can induce the maximal motor response. The charge distributions were also significantly different in terms of maximal tolerated charge ( $\mathrm{P}$ $<0.001)$. The post-hoc analysis revealed that charge distributions $\mathbf{a}$ and $\mathbf{b}$ reached significantly higher values of charge than distributions $\mathbf{d}$ and e. A previous study [30] 

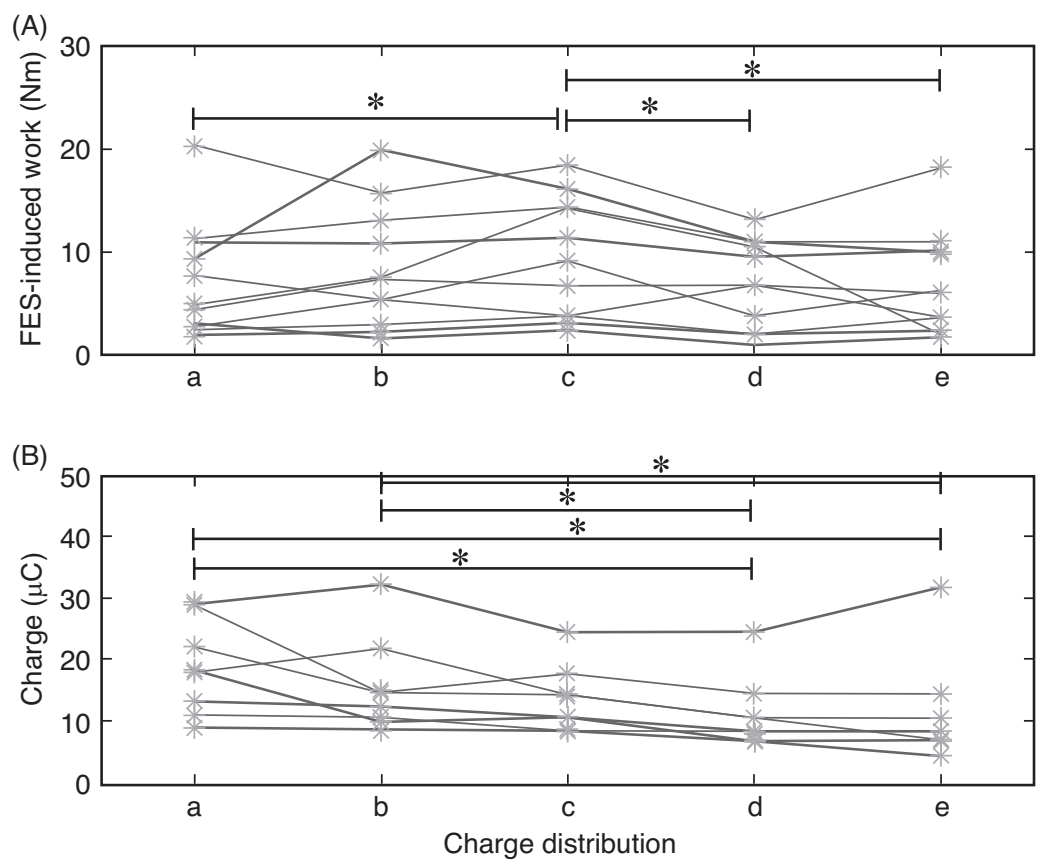

Figure 5. Comparison of the charge distributions in term of maximal FES-induced work (panel (A)) and maximal tolerated charge (panel (B)). Values obtained by all subjects and muscles not affected by fatigue are reported. $*$ indicates significant difference (Dunn-Sidak, $\mathrm{P}<0.05$ ).

investigated the relationship between stimulus parameters and muscle force, and reported that in intramuscular electrical stimulation, given a certain amount of charge, stimuli modulated in pulse width with a fixed amplitude produced higher muscle force than stimuli modulated in amplitude with a fixed PW. Our experiments partly confirmed these results. Indeed, charge distribution $\mathbf{c}$ produced in most cases higher work than charge distributions $\mathbf{a}\left(\mathrm{PW}_{\mathrm{a}}=500 \mu \mathrm{s}\right)$ and $\mathbf{b}\left(\mathrm{PW}_{\mathrm{b}}>\mathrm{PW}_{\mathrm{c}}, \mathrm{A}_{\mathrm{b}}<\mathrm{A}_{\mathrm{c}}\right)$ although lower values of charge were reached. Theoretically, distributions $\mathbf{d}\left(\mathrm{PW}_{\mathrm{d}}<\mathrm{PW}_{\mathrm{c}}, \mathrm{A}_{\mathrm{d}}>\mathrm{A}_{\mathrm{c}}\right)$ and $\mathbf{e}\left(\mathrm{A}_{\mathrm{e}}=70 \mathrm{~mA}\right)$ would have been able to produce even higher values of work. However, in transcutaneous electrical stimulation, the pain threshold plays a crucial role, and when distributions $\mathbf{d}$ and $\mathbf{e}$ were used, the subjects were able to tolerate lower values of maximal charge. This suggests that, given a certain amount of charge, stimuli with higher values of PW and lower values of amplitude are more tolerated but produce lower muscle force. Thus, charge distribution c, characterized by an intermediate behavior in terms of modulation of current and PW, maximized the motor output within the tolerance constraint and was selected to be tested on patients. Maximizing the motor output lead to maximization of the FES-induced peripheral benefits, such as the increase in muscle strength, the enhancement of cardiorespiratory fitness, the improvement in tissue oxygenation and peripheral hemodynamic function [31]. 


\subsection{Feasibility Trials on Patients}

Figure 6 shows the results obtained by S7 during the trial for identifying the stimulation strategy. The left side was stimulated since the subject was affected by a right hemiparesis following a traumatic brain injury (see Table 1). Analogous values of FESinduced active torque were produced by the patient with respect to the healthy subjects (see Figure 3 for comparison). The resultant stimulation ranges were considered biomimetic; indeed, an overlap of $100 \%$ between the estimated stimulation ranges and the physiological muscular strategy was found for all muscles.

Table 2 reports the results obtained from patients both in terms of session-specific stimulation ranges and overlap values. The procedure was able to identify a biomimetic stimulation strategy on 6 out of 8 patients. For S2 and S5, an overlap of $80 \%$ was not achieved for all muscles and thus, the standard stimulation strategy was used. The last raw of Table 2 reports the standard stimulation ranges defined in [16] for comparison. Many reasons might explain why the procedure was not successful for these 2 patients. It is likely that the patients were not able to relax during the trial and voluntarily
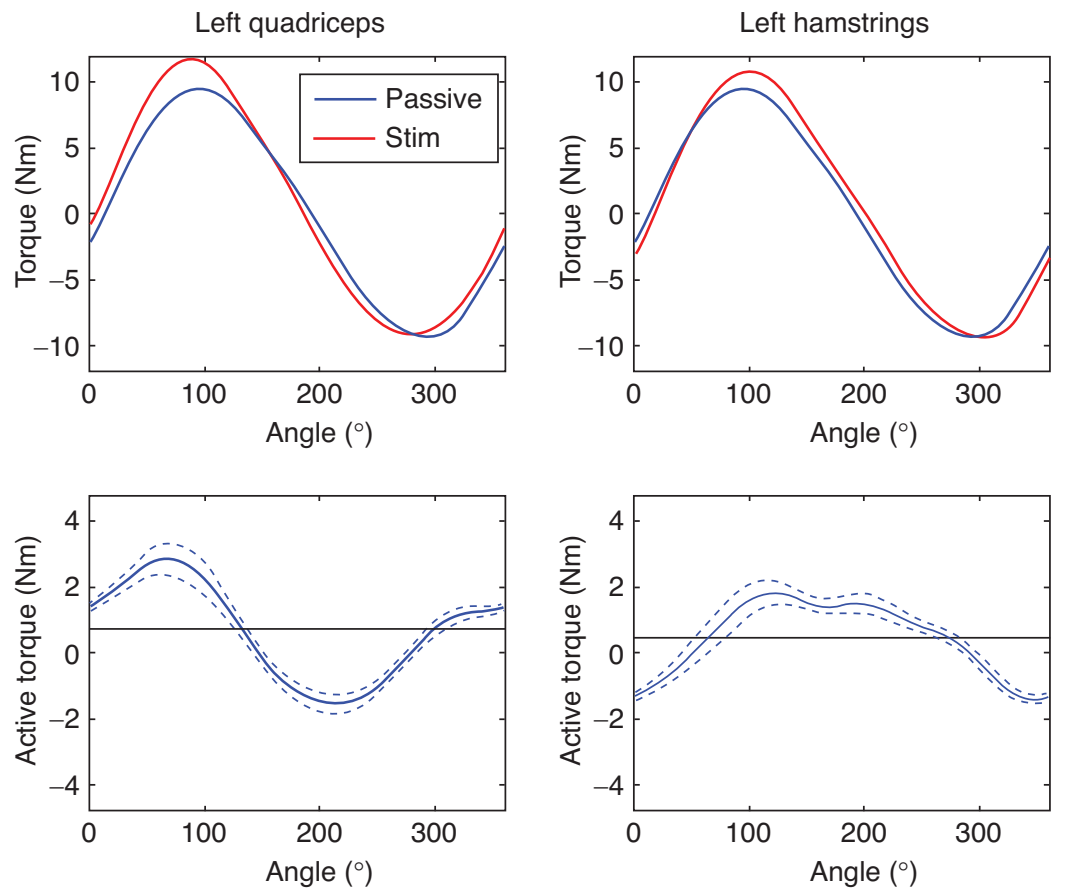

Figure 6. Results of the trial to identify the stimulation strategy on patient S7. Panels (A) and (B) depict the mean torques during passive cycling and stimulation phases of the left (unimpaired) quadriceps and hamstrings, respectively; panels (C) and (D) report the corresponding FES-induced active torques: the solid line shows the mean profiles, the dashed lines represent the SD, and the black horizontal line indicates the threshold used to identify the static stimulation ranges. 
Table 2. Results of the session-specific stimulation strategy obtained from patients.

\begin{tabular}{|c|c|c|c|c|c|c|c|c|}
\hline \multirow[b]{2}{*}{ Subject } & \multicolumn{4}{|c|}{ Static stimulation ranges $\left[^{\circ}\right]$} & \multicolumn{4}{|c|}{ Overlap [\%] } \\
\hline & $\begin{array}{l}\text { Right } \\
\text { Quad }\end{array}$ & $\begin{array}{l}\text { Right } \\
\text { Ham }\end{array}$ & $\begin{array}{c}\text { Left } \\
\text { Quad }\end{array}$ & $\begin{array}{l}\text { Left } \\
\text { Ham }\end{array}$ & $\begin{array}{l}\text { Right } \\
\text { Quad }\end{array}$ & $\begin{array}{l}\text { Right } \\
\text { Ham }\end{array}$ & $\begin{array}{l}\text { Left } \\
\text { Quad }\end{array}$ & $\begin{array}{l}\text { Left } \\
\text { Ham }\end{array}$ \\
\hline S1 & $147-260$ & $321-48$ & $327-80$ & $141-228$ & 100 & 100 & 100 & 100 \\
\hline S2 & $13-312$ & $69-240$ & $193-132$ & $249-60$ & 72 & 47 & 75 & 30 \\
\hline S3 & $196-262$ & $311-73$ & $16-82$ & $131-253$ & 100 & 100 & 100 & 100 \\
\hline S4 & $137-303$ & $280-42$ & $317-123$ & $100-222$ & 100 & 100 & 99 & 100 \\
\hline S5 & $347-138$ & $336-136$ & $167-318$ & $156-316$ & 28 & 86 & 40 & 71 \\
\hline S6 & $142-306$ & $3-93$ & $322-126$ & $183-273$ & 100 & 100 & 97 & 91 \\
\hline S7 & $120-293$ & $277-55$ & $300-113$ & $97-235$ & 100 & 100 & 100 & 100 \\
\hline S8 & $166-285$ & $287-60$ & $346-105$ & $107-240$ & 100 & 100 & 100 & 100 \\
\hline Standard & $173-303$ & $303-73$ & $353-123$ & $123-253$ & 100 & 100 & 100 & 100 \\
\hline
\end{tabular}

contributed to the movement but in a non-physiological manner so as to alter the FESinduced active torque measured at the crank. It may be also possible that a higher stimulation amplitude was required to induce a positive active torque at the crank but the patients were not able to tolerate higher values. This could happen quite often in hemiparetic patients because of muscular atrophy combined with an unaltered perception level. Some sessions of FES-induced exercises could be helpful to increase the muscular tone and to diminish the discomfort induced by FES (habituation effect); afterwards, the setting of the session-specific stimulation strategy might be more effective.

The entire procedure lasted less than five minutes that can be considered an acceptable duration if compared to the standard length of an FES-cycling training session (about 30 minutes $[1,4,7]$ ).

\subsection{Limitations of the Study and Future Directions}

This study describes a novel method that could promote the clinical acceptance of FEScycling training. However, there are some limitations to point out.

Healthy subjects and hemiparetic patients were asked to relax during the experiments but they could have contributed somehow to the movement, altering the FES-induced torque. To check whether the subjects were actually relaxed, the active torque profiles were analyzed. A high repeatability and a tight correlation with the stimulation phase were checked to test the reliability of the data. However, a volitional contribution, either intentionally or non-intentionally, repeatable and synchronously summed to the stimulation cannot be completely excluded.

The best charge distribution was selected based on trials involving only young healthy subjects. However, potential differences between young healthy subjects and hemiparetic patients, such as muscular atrophy, fiber distribution type, or sarcopenia, could affect the muscular properties with a potential influence on the modulation of the stimulation parameters [32]. 
Although the rapid onset of muscular fatigue during FES-induced exercises limits the duration and the efficacy of the training, the effect of different parameters on muscular fatigue was not investigated and a low constant value of stimulation frequency $(20 \mathrm{~Hz})$ was used. This frequency, although limiting the torque produced at the crank, increased the possibility that a certain level of muscular contraction was maintained throughout the training [1,21] and allowed the use of the procedure in clinical settings. Indeed, the current commercially available stimulators for FEScycling do not allow modulation of the stimulation frequency during training. Furthermore, we did not investigate whether the identified stimulation parameters were still the best solution after the onset of muscular fatigue.

The proposed procedure requires the measurement of the force/torque signals at the two pedals [25]. However, commercial cycle-ergometers typically provide only the motor torque, a signal related to the total torque produced by both legs at the crank. To measure the contribution of each leg during pedaling, the ergometer can be equipped with a commercial bicycle instrument that enables pedal forces as well as power output measurements with a free choice of the pedal system [33].

Finally, to estimate a reliable set of parameters, a subject has to completely relax during the trials and be able to produce a significant amount of FES-induced torque. However, hemiparetic patients are typically characterized by muscular atrophy combined with an altered perception level, and both conditions might hinder the reliability of their stimulation strategy. Some sessions of FES-induced exercises aimed at increasing the muscular tone and allowing the patient to become familiar with FES might increase the possibility to automatically estimate of a biomimetic stimulation strategy.

\section{CONCLUSIONS}

FES-induced cycling training may be a useful intervention for hemiparetic patients, since it integrates different elements of the motor relearning process, such as repetition, functional goal-directed activity, and electrical stimulation. A novel automatic procedure to identify the session-specific stimulation parameters required during the training (i.e., the stimulation strategy and the pulse amplitude and duration of each stimulated muscle) was designed and its feasibility was assessed on eight patients. The proposed procedure is fast (lasting less than 5 minutes) and easy to use in a clinical environment. It can be performed before the beginning of every training session, and it is completely independent of the operator, the electrodes placement, and the position of the patient with respect to the ergometer. An experimental protocol, which can automatically estimate a biomimetic stimulation strategy, and a method to optimize the motor output within the tolerance constraint were developed. This approach might further enhance the therapeutic effects of FES-cycling training, both helping in relearning a physiological muscular activation strategy during a functional and coordinated movement and enhancing the peripheral benefits induced by FES. In conclusion, such a procedure might enhance the training performance, simultaneously reducing the set-up time, so as to promote the clinical uptake of FES-cycling training. 


\section{ACKNOWLEDGEMENTS}

This work was supported by the Italian Ministry of Education, Universities and Research (grant no.: 2010R277FT, project title "Fall risk estimation and prevention in the elderly using a quantitative multifactorial approach"), by the Italian Ministry of Healthy (Fondazione Maugeri, grant no.: GR-2010-2312228, project title "Fall prevention and locomotion recovery in post-stroke patients: a multimodal training towards a more autonomous daily life"), and the German Federal Ministry of Education and Research (project RehaRobES, grant no.: FKZ 01EZ0766).

\section{CONFLICT OF INTERESTS}

The authors do not have any direct financial relation with the commercial identities mentioned in the publication that might lead to a conflict of interest and there has been no significant financial support for this work that could have influenced its outcome.

\section{REFERENCES}

[1] Ferrante S, Pedrocchi A, Ferrigno G, Molteni F. Cycling induced by functional electrical stimulation improves the muscular strength and the motor control of individuals with post-acute stroke. Europa Medicophysica-SIMFER 2007 Award Winner. Eur J Phys Rehabil Med, 2008, 44(2):159-67.

[2] Szecsi J, Krewer C, Müller F, Straube A. Functional electrical stimulation assisted cycling of patients with subacute stroke: kinetic and kinematic analysis. Clin Biomech (Bristol, Avon), 2008, 23(8):1086-94.

[3] Ambrosini E, Ferrante S, Schauer T, Ferrigno G, Molteni F, Pedrocchi A. Design of a symmetry controller for cycling induced by electrical stimulation: preliminary results on post-acute stroke patients. Artif Organs, 2010, 34(8):663-7.

[4] Ambrosini E, Ferrante S, Pedrocchi A, Ferrigno G, Molteni F. Cycling induced by electrical stimulation improves motor recovery in postacute hemiparetic patients: a randomized controlled trial. Strok,. 2011 Apr, 42(4):1068-73.

[5] Ambrosini E, Ferrante S, Ferrigno G, Molteni F, Pedrocchi A. Cycling induced by electrical stimulation improves muscle activation and symmetry during pedaling in hemiparetic patients. IEEE Trans Neural Syst Rehabil Eng, 2012, 20(3):320-30.

[6] Janssen TW, Beltman JM, Elich P, Koppe PA, Konijnenbelt H, de Haan A, et al. Effects of electric stimulation-assisted cycling training in people with chronic stroke. Arch Phys Med Rehabil, 2008, 89(3):463-9.

[7] Alon G, Conroy VM, Donner TW. Intensive training of subjects with chronic hemiparesis on a motorized cycle combined with functional electrical stimulation (FES): a feasibility and safety study. Physiother Res Int, 2011, 16(2):81-91.

[8] Braz GP, Russold M, Davis GM. Functional electrical stimulation control of standing and stepping after spinal cord injury: a review of technical characteristics. Neuromodulation, 2009 Jul, 12(3):180-90.

[9] Chen JJ, Yu NY, Huang DG, Ann BT, Chang GC. Applying fuzzy logic to control cycling movement induced by functional electrical stimulation. IEEE Trans Rehabil Eng, 1997, 5(2):158-69.

[10] Gföhler M, Lugner P. Dynamic simulation of FES-cycling: influence of individual parameters. IEEE Trans Neural Syst Rehabil Eng, 2004, 12(4):398-405.

[11] Hunt KJ, Stone B, Negård N-O, Schauer T, Fraser MH, Cathcart AJ, et al. Control strategies for integration of electric motor assist and functional electrical stimulation in paraplegic cycling: utility for exercise testing and mobile cycling. IEEE Trans Neural Syst Rehabil Eng, 2004, 12(1):89-101. 
[12] Perkins, TA, Donaldson, N, Fitzwater, R, Phillips, GF, Wood, DE. Leg powered paraplegic cycling system using surface Functional Electrical Stimulation. Artif Organs, 2002, 26 (3) 297-298.

[13] Petrofsky JS. New algorithm to control a cycle ergometer using electrical stimulation. Med Biol Eng Comput, 2003, 41(1):18-27.

[14] Petrofsky JS, Phillips CA, Almeyda J, Briggs R, Couch W, Colby W. Aerobic Trainer with Physiological Monitoring for Exercise in Paraplegic and Quadriplegic Patients. Journal of Clinical Engineering, 1985, 10(4):307-16.

[15] Pons DJ, Vaughan CL, Jaros GG. Cycling device powered by the electrically stimulated muscles of paraplegics. Med Biol Eng Comput, 1989, 27(1):1-7.

[16] Ferrante, S, Pedrocchi, A, Ferrigno, G. Biomimetic neuroprostheses: Human-Like Control Strategies to Improve Training Rehabilitative Exercises Using Functional Electrical Stimulation. Progress in Biological Cybernetics Research. Daan A. De Jong, 2008.

[17] Sheffler LR, Chae J. Neuromuscular electrical stimulation in neurorehabilitation. Muscle Nerve, 2007, 35(5):562-90.

[18] Gorgey AS, Mahoney E, Kendall T, Dudley GA. Effects of neuromuscular electrical stimulation parameters on specific tension. Eur J Appl Physiol, 2006, 97(6):737-44.

[19] Mesin L, Merlo E, Merletti R, Orizio C. Investigation of motor unit recruitment during stimulated contractions of tibialis anterior muscle. J Electromyogr Kinesiol, 2010, 20(4):580-9.

[20] Bergquist AJ, Clair JM, Lagerquist O, Mang CS, Okuma Y, Collins DF. Neuromuscular electrical stimulation: implications of the electrically evoked sensory volley. Eur J Appl Physiol. 2011 Oct, 111(10):2409-26.

[21] Doucet BM, Lam A, Griffin L. Neuromuscular electrical stimulation for skeletal muscle function. Yale J Biol Med, 2012, 85(2):201-15.

[22] Chou L-W, Binder-Macleod SA. The effects of stimulation frequency and fatigue on the forceintensity relationship for human skeletal muscle. Clin Neurophysiol, 2007, 118(6):1387-96.

[23] De Kroon JR, Ijzerman MJ, Chae J, Lankhorst GJ, Zilvold G. Relation between stimulation characteristics and clinical outcome in studies using electrical stimulation to improve motor control of the upper extremity in stroke. J Rehabil Med, 2005, 37(2): 65-74.

[24] Pomeroy VM, King L, Pollock A, Baily-Hallam A, Langhorne P. Electrostimulation for promoting recovery of movement or functional ability after stroke. Cochrane Database Syst Rev, 2006, (2): CD003241

[25] Comolli L, Ferrante S, Pedrocchi A, Bocciolone M, Ferrigno G, Molteni F. Metrological characterization of a cycle-ergometer to optimize the cycling induced by functional electrical stimulation on patients with stroke. Med Eng Phys. 2010, 32(4):339-48.

[26] Ferrante S, Ambrosini E, Ravelli P, Guanziroli E, Molteni F, Ferrigno G, et al. A biofeedback cycling training to improve locomotion: a case series study based on gait pattern classification of 153 chronic stroke patients. J Neuroeng Rehabil, 2011, 8:47.

[27] Hermens HJ, Freriks B, Disselhorst-Klug C, Rau G. Development of recommendations for SEMG sensors and sensor placement procedures. J Electromyogr Kinesiol, 2000, 10(5):361-74.

[28] Sharma N, Gregory CM, Dixon WE. Predictor-based compensation for electromechanical delay during neuromuscular electrical stimulation. IEEE Trans Neural Syst Rehabil Eng, 2011, 19(6):601-11.

[29] Kautz SA, Brown DA. Relationships between timing of muscle excitation and impaired motor performance during cyclical lower extremity movement in post-stroke hemiplegia. Brain, 1998, 121 ( Pt 3):515-26.

[30] Crago PE, Peckham PH, Thrope GB. Modulation of muscle force by recruitment during intramuscular stimulation. IEEE Trans Biomed Eng, 1980, 27(12):679-84. 
[31] Glinsky J, Harvey L, Van Es P. Efficacy of electrical stimulation to increase muscle strength in people with neurological conditions: a systematic review. Physiother Res Int, 2007, 12(3):175-94.

[32] Ambrosini E, Ferrante S, Schauer T, Klauer C, Gaffuri M, Ferrigno G, Pedrocchi A. A myocontrolled neuroprosthesis integrated with a passive exoskeleton to support upper limb activities. Journal of Electromyography and Kinesiology. 2014, 24(2):307-17.

[33] Stapelfeldt B, Mornieux G, Oberheim R, Belli A, Gollhofer A. Development and evaluation of a new bicycle instrument for measurements of pedal forces and power output in cycling. Int J Sports Med, 2007, 28(4):326-32. 



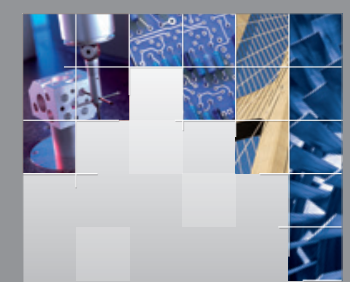

\section{Enfincering}
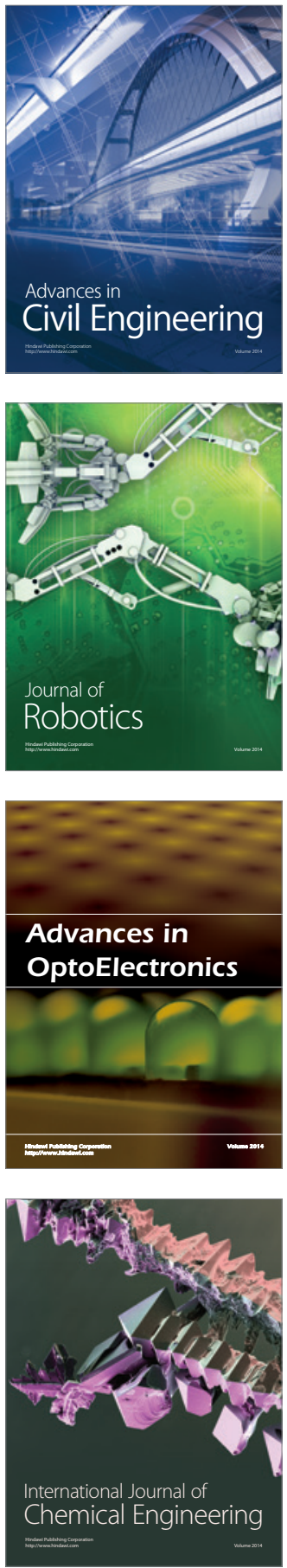

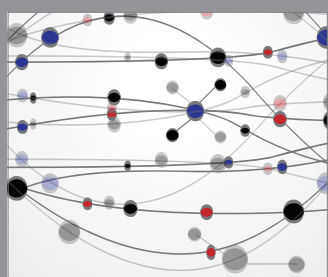

The Scientific World Journal

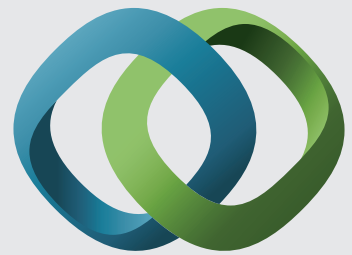

\section{Hindawi}

Submit your manuscripts at

http://www.hindawi.com
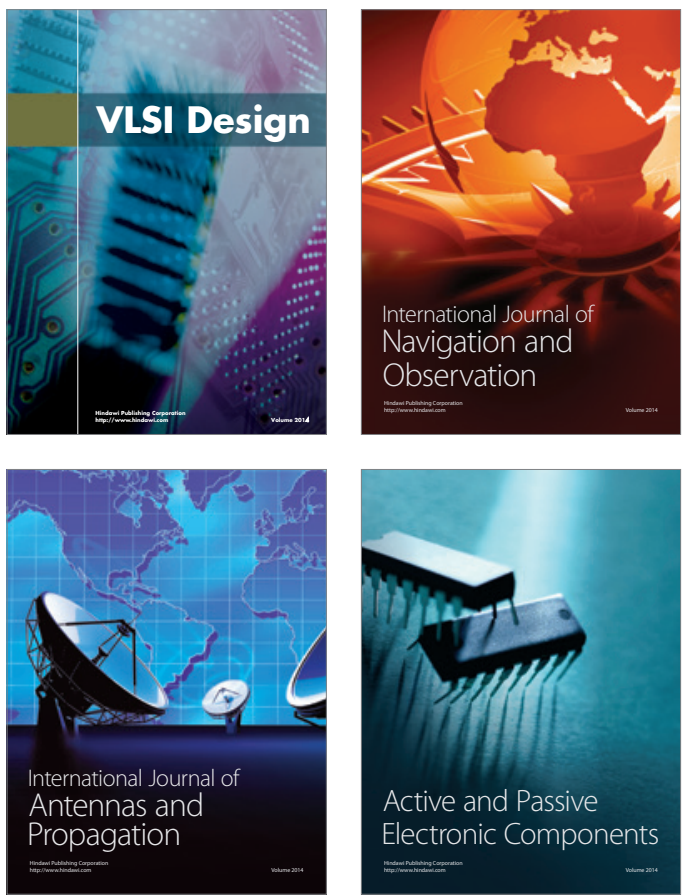
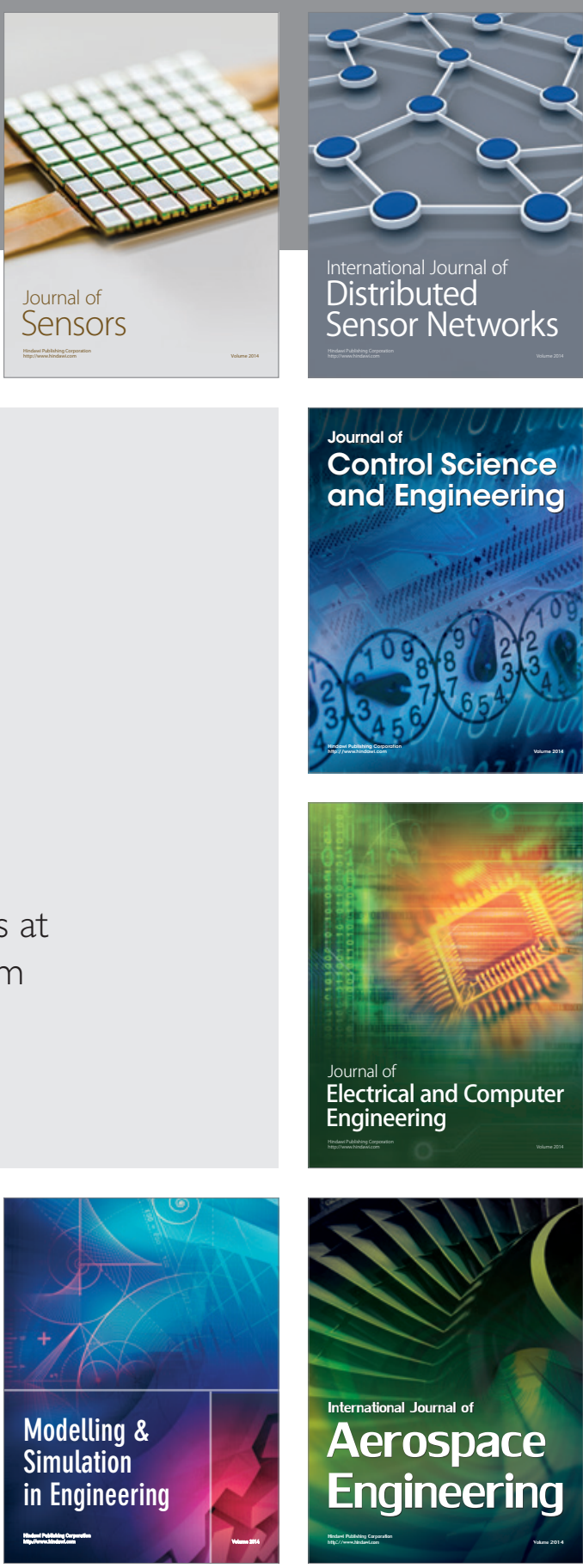

International Journal of

Distributed

Sensor Networks

Journal of

Control Science

and Engineering
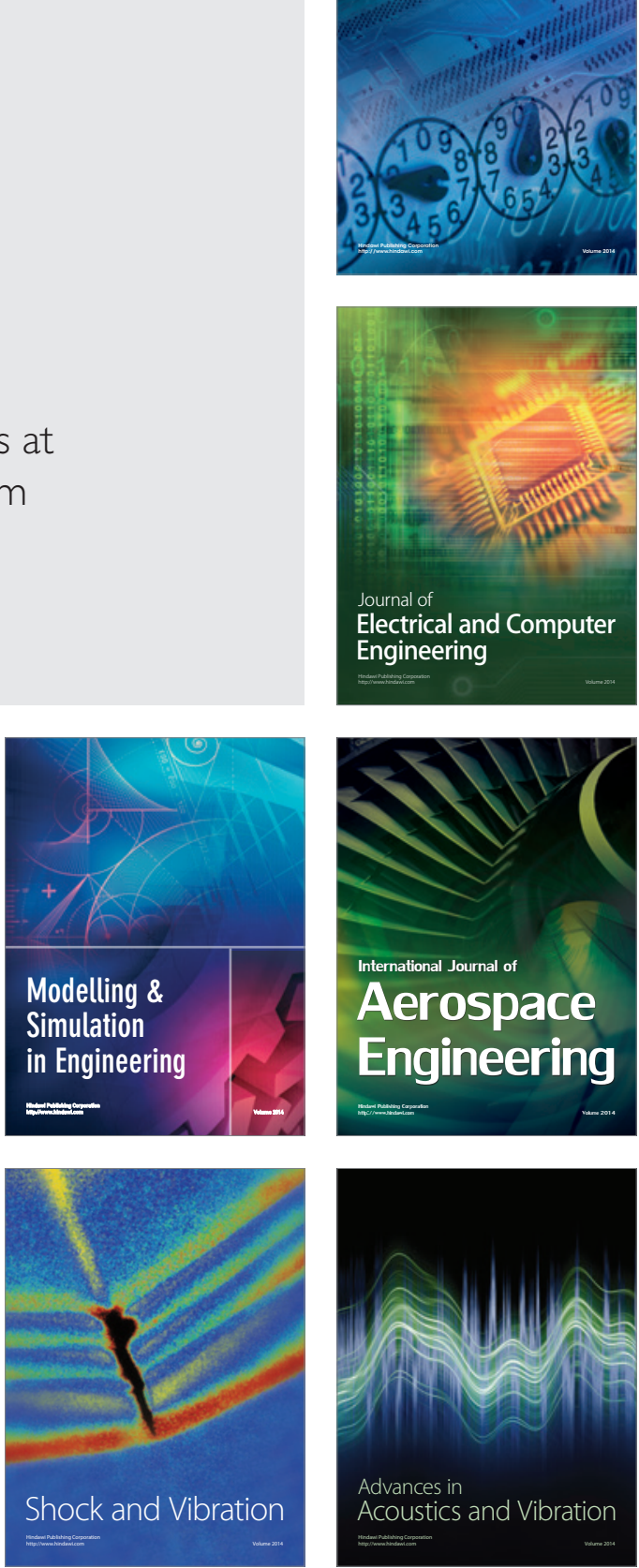